\title{
Short-Term Exposure to High-Temperature Water Causes a Shift in the Microbiome of the Common Aquarium Sponge Lendenfeldia chondrodes
}

\author{
Sergio Vargas $^{1}$ (D) Laura Leiva $^{1,2}$ (D) $\cdot$ Gert Wörheide ${ }^{1,3,4}$ (D) \\ Received: 14 January 2020 / Accepted: 8 July 2020 / Published online: 7 August 2020 \\ (C) The Author(s) 2020
}

\begin{abstract}
Marine sponges harbor diverse microbiomes that contribute to their energetic and metabolic needs. Although numerous studies on sponge microbial diversity exist, relatively few focused on sponge microbial community changes under different sources of environmental stress. In this study, we assess the impact of elevated seawater temperature on the microbiome of cultured Lendenfeldia chondrodes, a coral reef sponge commonly found in marine aquaria. Lendenfeldia chondrodes exhibits high thermal tolerance showing no evidence of tissue damage or bleaching at $5^{\circ} \mathrm{C}$ above control water temperature $\left(26^{\circ} \mathrm{C}\right)$. Highthroughput sequencing of the bacterial $16 \mathrm{~S}$ rRNA V4 region revealed a response of the microbiome of $L$. chondrodes to shortterm exposure to elevated seawater temperature. Shifts in abundance and richness of the dominant bacterial phyla found in the microbiome of this species, namely Proteobacteria, Cyanobacteria, Planctomycetes, and Bacteroidetes, characterized this response. The observed resilience of $L$. chondrodes and the responsiveness of its microbiome to short-term increases in seawater temperature suggest that this holobiont may be capable of acclimating to anthropogenic-driven sublethal environmental stress via a re-accommodation of its associated bacterial community. This sheds a new light on the potential for resilience of some sponges to increasing surface seawater temperatures and associated projected regime shifts in coral reefs.
\end{abstract}

Keywords Climate change $\cdot$ Cyanobacteria $\cdot$ Lendenfeldia chondrodes $\cdot$ Microbiome $\cdot$ Sponges $\cdot$ Porifera

\section{Introduction}

By the end of this century, moderate climate change scenarios predict the surface temperature of the globe to be over $1.5^{\circ} \mathrm{C}$

Electronic supplementary material The online version of this article (https://doi.org/10.1007/s00248-020-01556-z) contains supplementary material, which is available to authorized users.

Sergio Vargas

sergio.vargas@1mu.de

1 Department of Earth and Environmental Sciences, Paleontology \& Geobiology, Ludwig-Maximilians-Universität München, Richard-Wagner-Str. 10, 80333 Munich, Germany

2 Present address: Biologische Anstalt Helgoland, Shelf Sea System Ecology, Alfred-Wegener-Institut Helmholtz-Zentrum für Polar- und Meeresforschung, 27498 Helgoland, Germany

3 GeoBio-Center, Ludwig-Maximilians-Universität München, Richard-Wagner-Str. 10, 80333 Munich, Germany

4 SNSB - Bayerische Staatssammlung für Paläontologie und Geologie, Richard-Wagner-Str. 10, 80333 Munich, Germany higher than in 1850-1900, and the oceans are expected to keep warming throughout the twenty-first century [1]. Extreme events, such as the marine heatwave recorded on the Great Barrier Reef in 2016, are expected to become more common under those scenarios [1], and their detrimental impact on coastal ecosystems will undoubtedly continue to cause significant shifts in the species composition of marine communities [e.g., 2].

An increase in seawater temperature of one to two degrees over maximum summer temperatures approaches the upper tolerance level of important coastal ecosystem engineers, like corals [3] and some sponges tested to date [4-9]. The reef sponge Rhopaloeides odorabile, for instance, showed signs of necrosis after $24 \mathrm{~h}$ of exposure to seawater temperatures of $33^{\circ} \mathrm{C}$, only about two to four degrees above the mean highest summer temperature at the site of collection [4]. At this temperature, the bacterial community associated with $R$. odorabile suffers irreversible compositional shifts likely caused by a "breakdown" of the sponge symbiotic functions and the colonization of the sponge by opportunistic, potentially pathogenic bacteria [4]. Simister et al. [10] reported similar results. These authors found a shift in the bacterial community associated with $R$. odorabile 
after exposing this sponge to a seawater temperature of $32^{\circ} \mathrm{C}$. In both experiments, the authors reported a highly stable microbiome at temperatures below that upper limit $[4,10]$.

Beside $R$. odorabile, several reports of a highly stable sponge microbiome at temperatures below a specific threshold exists. The microbiome of the Australian reef sponge Ianthella basta is stable across a latitudinal gradient of over $1000 \mathrm{~km}$ and at temperatures up to $31^{\circ} \mathrm{C}$, with microbiome changes only evident after exposure of the sponges to lethal water temperatures (> $32{ }^{\circ} \mathrm{C}$ ) [5]. Also, the microbiome of the Mediterranean species Ircinia fasciculata and I. oros appears to be resistant to sublethal heat stress [11]. However, once the water temperature exceeds an upper limit, the microbiome of I. fasciculata changes, usually preceding the onset of disease and, eventually, the death of the affected sponges [9]. Perhaps unexpectedly, the microbiome of the boreal sponge Geodia barreti was also not affected by exposure to acute thermal stress (i.e., $5{ }^{\circ} \mathrm{C}$ above "normal" environmental seawater temperature) for up to 14 days [12]. Thus, the resilience of the sponge microbiome to sublethal thermal stress does not appear to be determined by the geographic area where the sponges occur.

Throughout its $\sim 600$ million years long evolutionary history $[13,14]$, Phylum Porifera has evolved to become a significant part of benthic communities worldwide [15], occurring from the tropics to the polar regions in habitats ranging from the eulittoral to the hadal zone [15-17] and playing essential roles in nutrient cycling in these ecosystems [15, 18-20]. The diverse functional roles and ecological flexibility of sponges partly result from their diverse microbiomes [21-23], and although many sponge species have highly stable microbiomes regardless of the environment [24, 25], environmental changes can trigger compositional shifts in sponge microbiomes [26] that can affect the fitness of these holobionts. Thus, investigating the effect of environmental change on the sponge microbiome is pivotal to our understanding of the consequences of climate change upon sponge holobionts and the benthic communities where these organisms occur.

Lendenfeldia chondrodes, a common (blue) aquarium sponge, is an easy-to-culture, promising (cyano)sponge holobiont model [27]. Here, we characterized the microbiome of $L$. chondrodes using high-throughput $16 \mathrm{~S}$ rRNA sequencing and examined the effect of a rapid, short-term increase in sea surface temperature (SST) on the diversity and community composition of the microbiome of this species.

\section{Material and Methods}

\section{General Description of the Aquarium System and the Heat Shock Experiments}

We cultured purple and green morphs of L. chondrodes (Suppl. Fig. 1) in a 360-L marine aquarium at the Molecular
Geobiology \& Paleobiology Laboratory of the Dept. of Earth and Environmental Sciences of the Ludwig-MaximiliansUniversität München, under a $12 \mathrm{~h}$ day, $12 \mathrm{~h}$ night cycle controlled by GHL Mitras LX 6200-HV LED lights. Light intensity at the water surface is $\sim 10$ klux. Based on hourly measurements over one year (2017), average water temperature and $\mathrm{pH}$ are $24.92 \pm 0.24^{\circ} \mathrm{C}$ and $8.30 \pm 0.14$, respectively. Based on weakly measurements over one year (2017), the average $\mathrm{PO}_{4}{ }^{3-}, \mathrm{NO}_{2}{ }^{-}$, and $\mathrm{NO}_{3}{ }^{-}$concentrations in the water are $0.092 \pm 0.071 \mathrm{mg} / \mathrm{L}, 0.014 \pm 0.072 \mathrm{mg} / \mathrm{L}$, and $2.681 \pm$ $3.882 \mathrm{mg} / \mathrm{L}$; the concentration of $\mathrm{NH}_{3} / \mathrm{NH}_{4}{ }^{+}$in the water was consistently below detection (i.e., $<0.05 \mathrm{mg} / \mathrm{L}$ ). These values are stable in time as shown by continued water sampling.

For the heat shock experiments, we cut circular sections of the sponges (12 per color morph) with a medical biopsy puncher $(0.5 \mathrm{~cm}$ diameter $)$ and let them heal in the main aquarium (see above) for one week. We then randomly distributed the sponge explants in six 10-L experimental tanks (i.e., two explants per color morph per tank) filled with $\sim 6 \mathrm{~L}$ artificial seawater and partially immersed in the 360-L aquarium described above. Water evaporation in the 10-L tanks was compensated every day with water filtered by reverse osmosis. To provide an adequate water mixing, we used a submersible water pump in each tank (300 L/h; Eheim, Germany). After an acclimation period of four days in the experimental tanks, we randomly selected three tanks and used a TetraTec HT 25 heating device (Eheim, Germany) to gradually increased the water temperature over a period of five days $(\sim$ $1{ }^{\circ} \mathrm{C}$ per day) from $\sim 25.4$ to $\sim 31{ }^{\circ} \mathrm{C}$. During the course of the experiment, we monitored the water temperature every minute using PCE-PHD 1 dataloggers (PCE Instruments, Germany) and, additionally, we manually measured the temperature of all aquaria twice a day (in the morning and the evening) using a regular thermometer (TFA, accuracy $\pm 0.5^{\circ} \mathrm{C}$ ). Throughout the experiment, water conductivity, $\mathrm{pH}$, density, redox potential, and nutrient profile in the 10-L tanks were similar to the values observed for the main tank. The water temperature of the control tanks was $\sim 25.4{ }^{\circ} \mathrm{C}$ over the whole experimental period. At the end of the experiment, we rinsed all explants with sterile-filtered artificial seawater, fixed them in $99 \%$ ethanol, and kept them at $-20{ }^{\circ} \mathrm{C}$ until further processing.

\section{High-Throughput Sequencing Bacterial 16S rRNA V4 Region of Control and Treated $L$. chondrodes}

We extracted genomic DNA using the Macherey-Nagel NucleoSpin DNA extraction kit, following the protocol provided by the manufacturer, and amplified the V4 region of the 16S rRNA gene using barcoded forward $(515 \mathrm{fB})$ and reverse (806rB) primers as described in [28]. In addition to the aquarium samples, and for comparison purposes only, we also sequenced a single specimen casually collected off at St John's 
Island, Singapore, at 3-m depth, and determined to be L. chondrodes. Before sequencing, we visualized PCR products on $1 \%$ agarose gels and extracted bands of the expected size (ca. 380 bp) with the Qiagen QIAquick Gel Extraction Kit. We then quantified all gel-extracted PCR products on a Qubit 3.0 fluorometer (Life Technologies, Grand Island, NY) and diluted them, if necessary, to achieve a final concentration of $1 \mathrm{nM}$ before equimolar pooling. We sequenced the $16 \mathrm{~S}$ rRNA amplicon pool on an Illumina MiniSeq in mid-output 300PE mode [see 28 for details]. We also determined the bacterial load of each extraction using real-time quantitative PCR (RT-qPCR). For this, using the same V4 region of the 16S rRNA forward (i.e., 515fB) and reverse (i.e., 806rB) primers but without the barcode+Illumina adapter extension, we amplified an untreated $L$. chondrodes sample, gelextracted the resulting amplicon, and quantified it on a Qubit 3.0 fluorometer (Life Technologies, Grand Island, NY). We then estimated the number of V4 16S rRNA molecules per $\mu \mathrm{L}$ purified amplicon using the formula N_molecules = $\left(\right.$ ng_DNA $\left.* 6.022 \times 10^{23}\right) /\left(\right.$ amplicon_length* $\left.1 \times 10^{9} * 650\right)$ with the expected amplicon length (i.e., $380 \mathrm{bp}$ ). For the calculation of the bacterial load per sample, we used the quantified amplicon to generate a standard dilution series ranging from $10^{2}$ to $10^{8} \mathrm{~V} 416 \mathrm{~S}$ rRNA molecules/ $\mu \mathrm{L}$ and used this series as the standard curve of a qPCR absolute quantification. For quantification, we used a three-step PCR with an initial denaturation step of $95^{\circ} \mathrm{C}$ for $3 \mathrm{~min}$ followed by up to 40 cycles consisting of $30 \mathrm{~s}$ denaturation at $95^{\circ} \mathrm{C}, 30 \mathrm{~s}$ annealing at $50^{\circ} \mathrm{C}$, and $30 \mathrm{~s}$ extension at $72{ }^{\circ} \mathrm{C}$. We performed a melting analysis after all qPCRs and used technical duplicates for both samples and standard dilutions. We also included duplicated no template samples as negative controls. After qPCR, we estimated the primer efficiency for each sample and repeated samples with efficiencies lower than 1.8. We used the bacterial load of each sample to provide bacterial load "corrected," absolute OTU counts after amplicon sequencing (see below) and used the corrected datasets to corroborate the results obtained using the uncorrected dataset. Unless otherwise specified in the text, we used the uncorrected counts for all analyses presented in this study. The reads generated are publicly available at the European Nucleotide Archive under study accession number PRJEB35927.

\section{Bioinformatic Analysis of the 16S rRNA Amplicon Data}

After demultiplexing, we processed the resulting sequences using vsearch [29]. Briefly, we assembled each sequence pair allowing a minimum overlap of $45 \mathrm{bp}$, dereplicated within and across samples, removed singletons and chimeras, and clustered sequences at $97 \%$ similarity to derive a "raw" operational taxonomic unit (OTU) counts table. We then used the RTqPCR data on bacterial load to provide absolute counts per sample for each OTU in the OTU table. For this, briefly, we rescaled the obtained OTU counts to the total number of bacteria per $\mu \mathrm{L}$ DNA. We determined the taxonomic affiliation of all OTUs found in L. chondrodes using the SILVA database "Alignment, Classification and Tree Service" (accessed May 22, 2019) and used the OTU count tables and the taxonomy annotations to determine phylum- and OTU-level richness and abundance patterns, and to fit rank-abundance dominance curves to the bacterial community associated with L. chondrodes. We also determined the core bacterial community of $L$. chondrodes as those bacterial OTUs present in all sequenced replicates independently of the treatment applied to them.

To assess the beta-diversity of treated vs. control sponges, we used non-metric multidimensional scaling (NMDS) with the Bray-Curtis distance. We also tested for significant effects of heat stress on bacterial composition using canonical correspondence analysis (CCA) with treatment (i.e., control vs. heat stress), color morph (i.e., green vs. purple) and their interaction as response variables. In order to test for a differential response to heat stress of core vs. non-core OTUs, we conducted separate CCAs for these bacterial groups. To better understand the dynamics of the bacterial community in response to heat stress, we identified OTUs gained or lost as a result of the treatment. For core OTUs, we also calculated logfold changes in heated vs. control samples. Finally, we compared the representative 16S rRNA sequences of the core OTUs against the top 24 most abundant OTUs derived from the single "wild" L. chondrodes sample available to us.

We used $\mathrm{R}$ with the packages vegan [30] and DESeq2 [31] for the analyses and deposited all scripts and raw data tables used in the project repository (https://gitlab.lrz.de/cbas/ CBAS_16S).

\section{Results}

\section{The Microbiome of $L$. chondrodes Is Rich but Dominated by a Small Number of Bacterial Associates}

In total, we successfully extracted, amplified, and sequenced a total of 20 samples. These included 6 purple and 6 green control sponges and 5 purple and 3 green treated sponges. Using these samples, we found 1343 OTUs affiliated to 22 bacterial phyla associated with $L$. chondrodes. Phylum Proteobacteria had the highest OTU richness (431 OTUs), followed by Bacteroidetes (185 OTUs) and Planctomycetes (156 OTUs). The remaining 19 phyla had considerably less OTUs assigned to them. For instance, we found 61 and 42 OTUs belonging to the phyla Cyanobacteria and Firmicutes, respectively, and all remaining phyla had 25 or fewer OTUs (Fig. 1). Dominance patterns differed radically from those of richness, with Cyanobacteria dominating the community and accumulating $47 \%$ of all the sequenced reads. The phyla 
Proteobacteria and Bacteroidetes followed in abundance with $29 \%$ and $13 \%$ of the reads, respectively. The remaining 19 phyla accounted for $11 \%$ of all the sequenced reads (Fig. 1). The core bacterial community of $L$. chondrodes is composed of 17 bacterial OTUs belonging to the phyla Acidobacteria, Actinobacteria, Bacteroidetes, Cyanobacteria, Planctomycetes, and Proteobacteria, and showing significant similarity to bacterial sequences derived from other sponges (Suppl. Table 1). These OTUs accounted for $78 \%$ of the sequenced reads across all samples. The analysis of a single L. chondrodes sample allowed us to compare the microbiome of cultured and wild L. chondrodes samples. In wild L. chondrodes, we found 62 bacterial OTUs of which the top 24 most abundant OTUs account for $99 \%$ of all the sequenced reads. Among these 24 OTUs, we found eight of the core OTUs detected in cultured L. chondrodes samples (Table 1). These OTUs had a 100\% identity between wild and cultured L. chondrodes. In addition, we found that the top two most abundant OTUs, namely one cyanobacterium and one bacteroidetes, in cultured and wild samples of L. chondrodes were identical.

As judged by its Akaike information criterion (AIC), the phylum-level best fit abundance-rank dominance model was a geometric series (niche preemption model; [32]) for 15 out of 20 replicates, followed by the Mandelbrot and the lognormal model in four and one replicates, respectively (Fig. 2). Fitting the Mandelbrot model to the phylum-level abundance data was challenging, and some parameters of this model could not be fit. Whenever the full model could be specified, the AIC for the Mandelbrot model was lower than that for the niche preemption model. Generally, the analysis of model deviance across replicates resulted in a non-significant difference between these models (Suppl. Table 2). At OTU-level, the best fit (i.e., lowest AIC) abundance-rank dominance models were the Zipf (15 replicates) or Mandelbrot (four replicates), with only one replicate fitting the lognormal model (Fig. 2). As in the analysis of the phylum-level abundances, an analysis of model deviance revealed that the Zipf and Mandelbrot models were not significantly different (Suppl. Table 3).

\section{Short-Term Exposure of $L$. chondrodes to Heat Stress Changes Core vs. Non-core Bacteria $\beta$-Diversity in a Specific Manner}

Heat stress caused a compositional change of the bacterial communities associated with $L$. chondrodes. This effect was somewhat mild in the NMDS plot (Fig. 3a) but marked in the canonical correspondence analysis, where control and treatment samples formed two distinct groups (Fig. 3b). Indeed, we found that the variable "Treatment" (i.e., heat stress vs. control temperature) had a significant effect on bacterial composition while the variable "Color morph" did not; the interaction between these two variables was not significant (Table 2). These results were independent of any filtering (e.g., including only OTUs with more than 50 reads) of the

\section{High-abundance phyla}

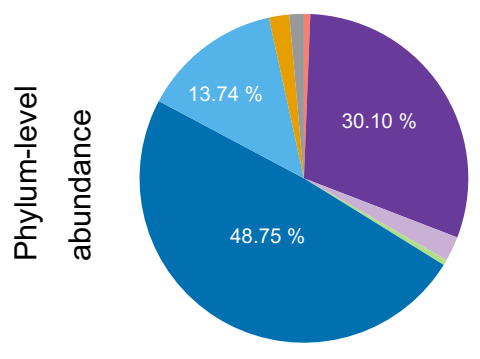

High-richness phyla

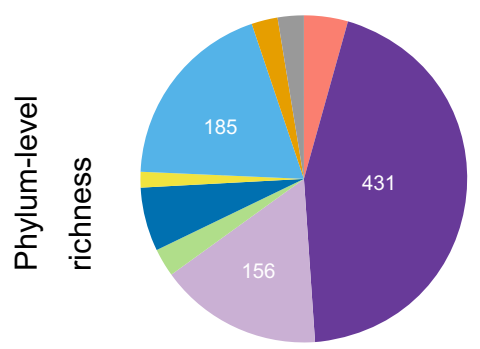

Fig. 1 Abundance and richness of bacterial phyla associated with the common aquarium sponge Lendenfeldia chondrodes. High-abundance phyla are defined as having at least 1000 16S V4 rRNA read counts. High-richness phyla are those with at least 10 OTUs. Supplementary

\section{Low-abundance phyla}

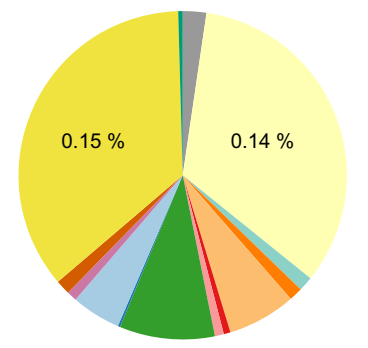

Low-richness phyla

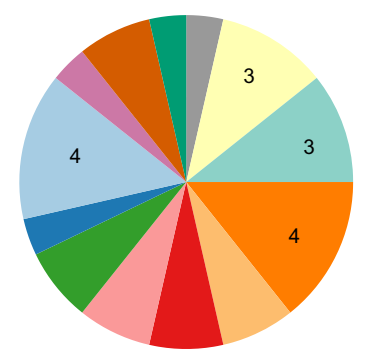

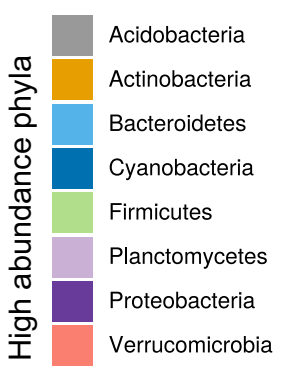

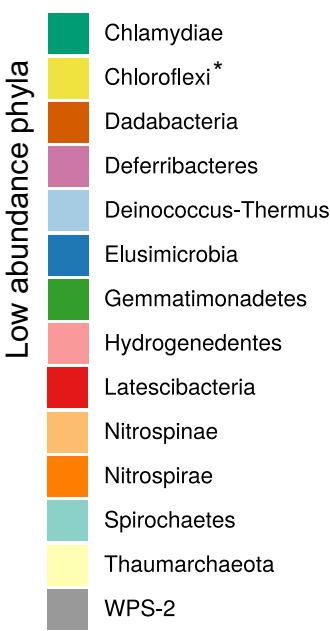

Figs. 2 and 3 provide phylum-level abundance and richness plots per sample, respectively. Rarefaction curves for each sample are provided in Suppl. Fig. 4 
Table 1 Core bacterial OTUs in wild vs. cultured samples of L. chondrodes. The OTU names are arbitrary and given by the OTUs clustering software independently for wild and cultured samples. OTU number roughly reflects the abundance rank, which is provided for wild and cultured samples. OTUs matching or with similar abundance ranks in bold. The taxonomic assignment was conducted independently for each sample and compared. Only OTUs with identical taxonomic assignment and $100 \%$ identical V4 16S rRNA sequences are reported

\begin{tabular}{|c|c|c|c|c|}
\hline \multicolumn{2}{|l|}{ OTUs } & \multicolumn{2}{|c|}{$\begin{array}{l}\text { Abundance } \\
\text { rank }\end{array}$} & \multirow[b]{2}{*}{ Taxonomy } \\
\hline Wild & Cultured & Wild & Cultured & \\
\hline OTU_1 & OTU_1 & 1 & 1 & Bacteria;Cyanobacteria;Oxyphotobacteria;Synechococcales;Cyanobiaceae;[Synechococcus] spongiarum group \\
\hline OTU_2 & OTU_2 & 2 & 2 & Bacteria;Bacteroidetes;Rhodothermia;Rhodothermales;Rhodothermaceae;uncultured \\
\hline OTU_6 & OTU_4 & 7 & 4 & Bacteria;Proteobacteria;Gammaproteobacteria;Oceanospirillales;Pseudohongiellaceae;Pseudohongiella \\
\hline OTU_4 & OTU_6 & 5 & 6 & Bacteria;Actinobacteria;Acidimicrobiia;Microtrichales;Microtrichaceae;Sva0996 marine group \\
\hline OTU_15 & OTU_11 & 18 & 8 & Bacteria;Acidobacteria;Subgroup 9 \\
\hline OTU_3 & OTU_18 & 3 & 10 & Bacteria;Proteobacteria;Gammaproteobacteria;Steroidobacterales;Woeseiaceae;JTB255 marine benthic group \\
\hline OTU_9 & OTU_21 & 10 & 11 & Bacteria;Proteobacteria;Gammaproteobacteria;UBA10353 marine group \\
\hline OTU_19 & OTU_59 & 22 & 15 & Bacteria;Proteobacteria;Alphaproteobacteria;Rhodovibrionales;Kiloniellaceae;uncultured \\
\hline
\end{tabular}

raw count OTU table we used for the analysis. However, these results differed from those we obtained using the bacterial load corrected OTU table. If we used these data, the NMDS analysis did not converge to a solution unless we filtered the OTU table to include only OTUs with more than 50 (absolute) counts. Using this (filtered) table, we also observed a significant effect of the variable "Treatment" on bacterial composition (Suppl. Table 4). In contrast to the analysis of the raw count table, we observed a significant effect of the variable "Color morph" on bacterial composition, while the interaction between these variables was still not significant (Suppl. Table 4).

The analysis of core and non-core bacterial OTUs revealed a similar trend and allowed us to better dissect the effect of heat stress on both types (i.e., core and non-core) of bacterial associates. Here again, we detected a significant effect of the heat stress treatment on both core and non-core bacterial associates. However, we only detected a significant effect of sponge color morph for non-core bacterial OTUs. Moreover, this group of OTUs was also affected by the interaction between thermal treatment and sponge color morph. In line with these results, we found that the heat stress treatment induced a change in the community characterized by the net loss of OTUs in treated vs. control sponges (Fig. 4).

Here, 11 out of 22 phyla detected in L. chondrodes loss OTUs, while only seven phyla gained new OTUs in response to heat stress. As expected, OTU replacement had a more notable impact on the richness of rare phyla, causing the disappearance of one bacterial phylum (Elusimicrobia) and lowering the richness of other bacterial phyla like Chloroflexi,
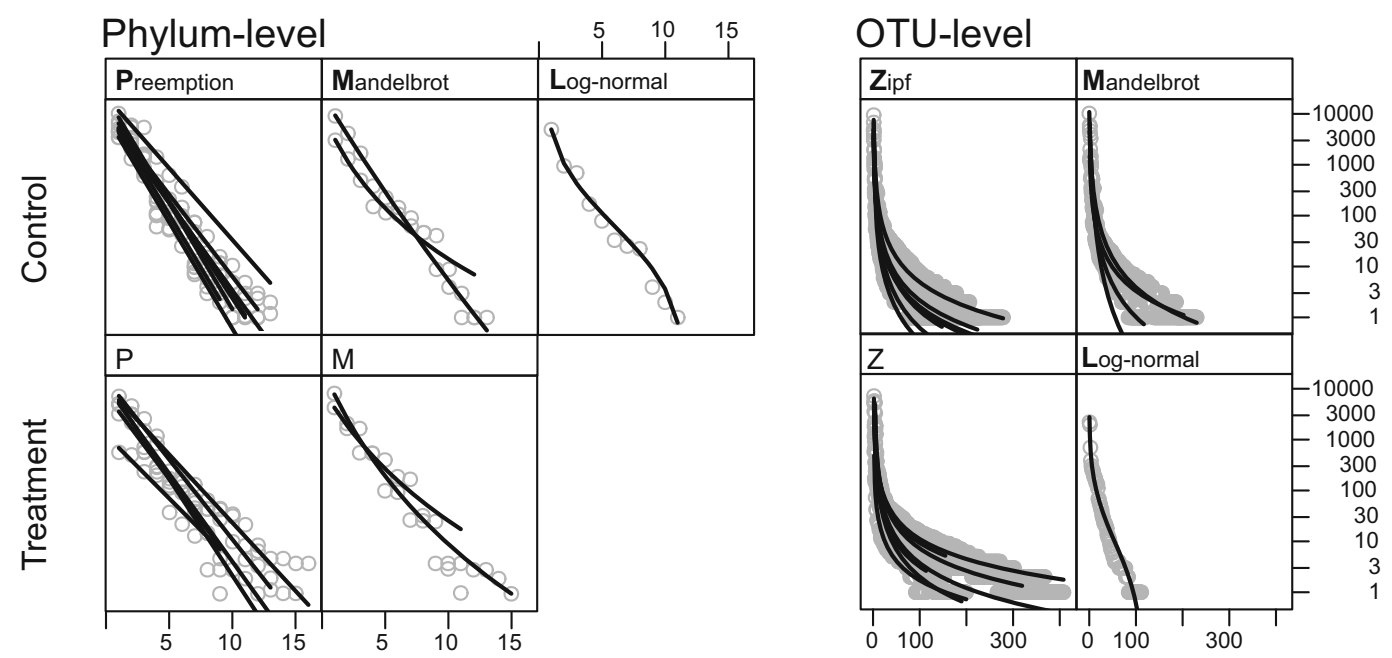

Rank

Fig. 2 Abundance-rank dominance models fitted to phylum- and OTU-level 16S V4 rRNA counts for control and thermal stress treatments. Lines represent sample replicates; dots are phyla/OTUs. The name of the models are above the curves, and the letter in bold is used as the model abbreviation 


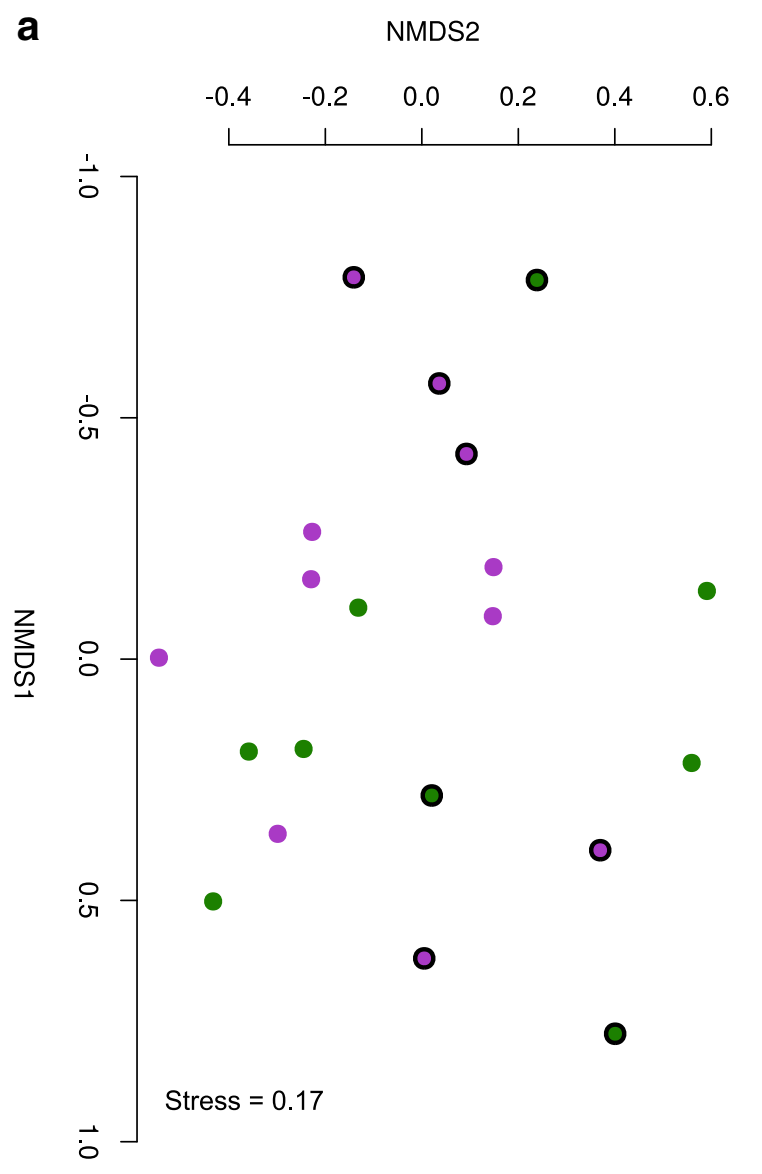

b

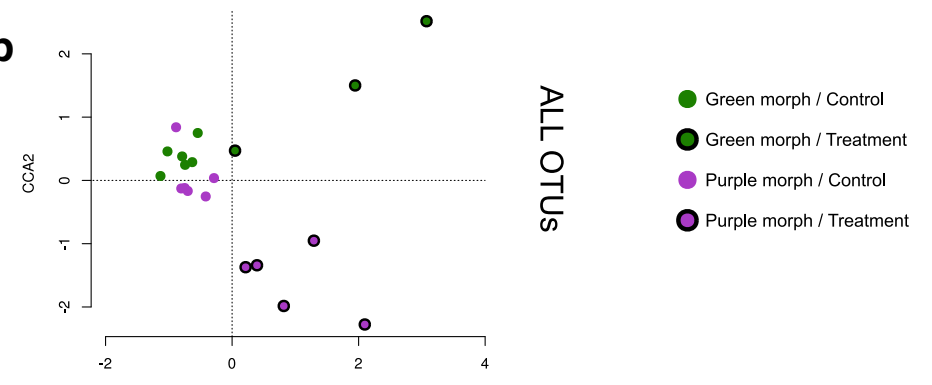

Fig. 3 Non-metric multidimensional scaling (a) and canonical correspondence (b) analysis of the OTU-level composition of the microbiome of Lendenfeldia chondrodes in control vs. thermal stress

Latescibacteria, Nitrospirae and, Spirochaetes in treated samples to less than half of the values detected in control sponges. Bacterial phyla benefiting from the heat stress treatment include Deferribacteres and Hydrogenedentes, which were only detectable in heat stress samples, and Deinococcus-Thermus and Nitrospinae, which duplicated their richness in treated vs. control sponges. Thaumarcheota also increased its richness in response to heat stress.

Table 2 Permutation test (999 permutations) for a canonical correspondence analysis of the effect of temperature treatment, sponge color morph, and their interaction on the composition of the microbiome of Lendenfeldia chondrodes. Terms added sequentially, first to last

\begin{tabular}{lrlll}
\hline & $d f$ & $\begin{array}{l}\text { Chi- } \\
\text { square }\end{array}$ & $F$ & $\operatorname{Pr}(>F)$ \\
\hline Treatment & 1 & 0.20026 & 1.7458 & 0.008 \\
Color morph & 1 & 0.11300 & 0.9850 & 0.492 \\
Treatment $\times$ color morph & 1 & 0.13395 & 1.1677 & 0.191 \\
Residual & 16 & 1.83543 & & \\
\hline
\end{tabular}

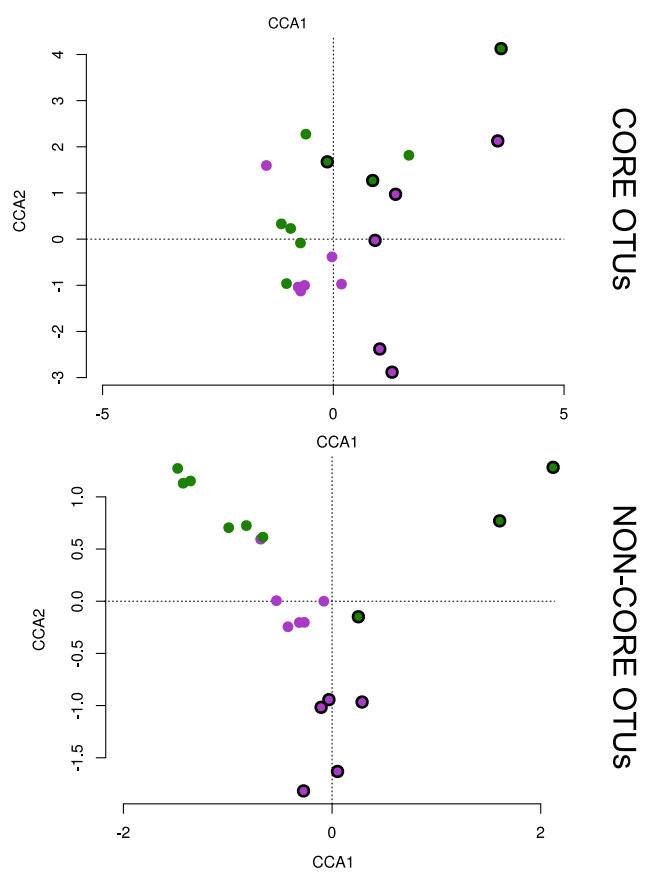

treatments. GC green morph control samples, PC purple morph control samples, GT green morph treatment samples, PT purple morph treatment samples

\section{Heat Stress Affects Different Members of the Core Bacterial Community Associated with $L$. chondrodes in a Specific Manner}

The analysis of abundance log-fold changes of L. chondrodes' 17 core bacterial OTUs revealed that heat stress had a specific effect on different members of this community. This differential response was not taxon-specific as the abundance of OTUs belonging to the same phylum responded in opposite ways upon exposure to heat stress (Fig. 5). For instance, within Proteobacteria, which with ten OTUs is the richest phylum in the core community of $L$. chondrodes, three OTUs halved (i.e., log-fold change less than -1) and four OTUs doubled (i.e., log-fold change $>1$ ) their abundances. This trend was also evident in the other phyla with more than one OTU, namely Bacteroidetes and Cyanobacteria. In both phyla, one OTU increased its abundance while the abundance of the remaining OTU assigned to the phylum decreased. The observed change in OTU abundance seems to correlate with the mean overall abundance of these OTUs (Fig. 5). This 
Fig. 4 Relative OTU gain/loss per phylum after exposure to thermal stress. Bacterial gain/loss is defined as the appearance/ disappearance of an OTU in the treated samples using as reference the composition of control samples

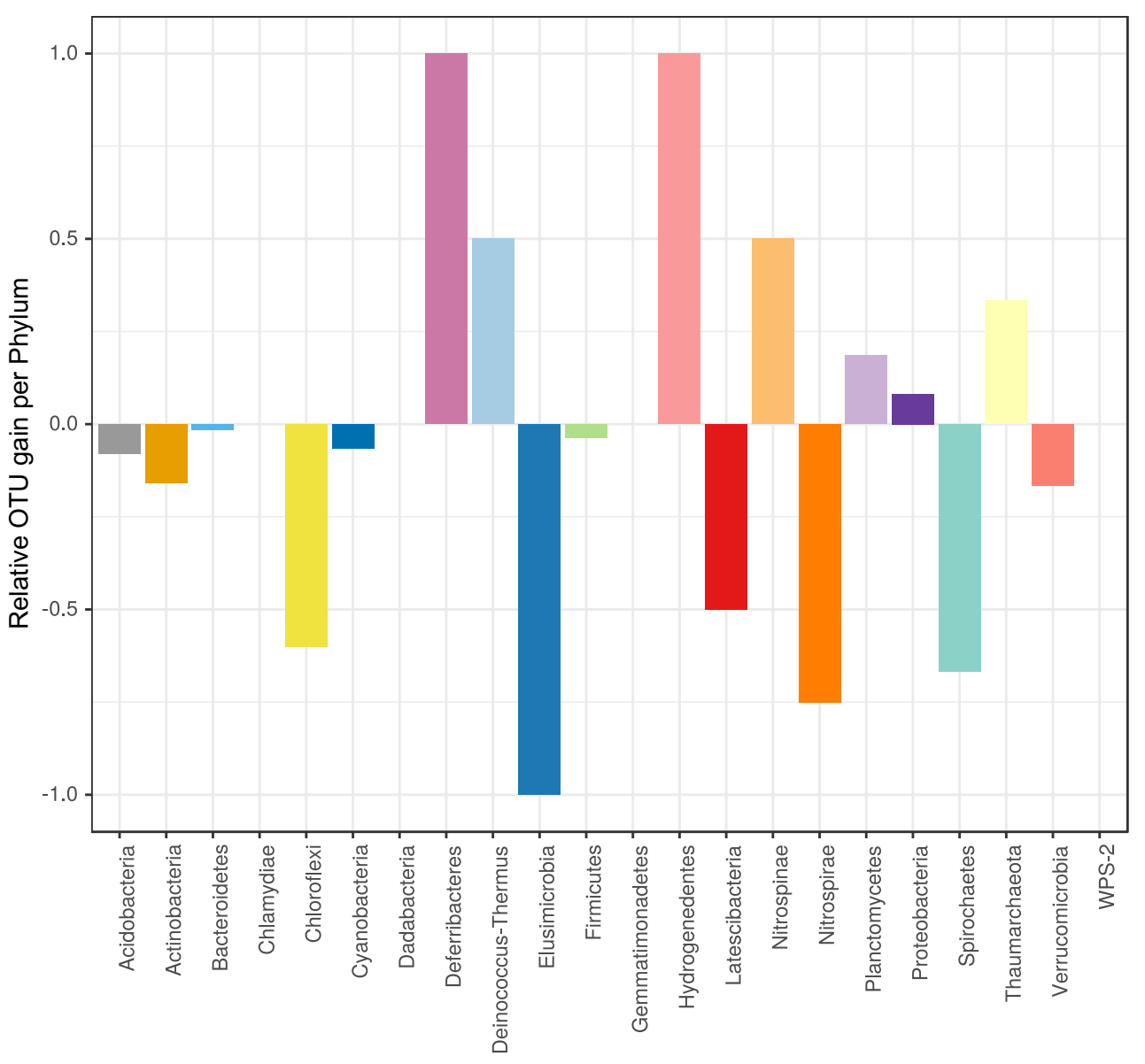

Phylum pattern appears to be general for the core bacterial community. Indeed, the four most and less abundant OTUs decreased and increased their abundance in response to heat stress. Despite this, we did not find a significant correlation between abundance log-fold change and mean overall OTU abundance. Exploration of the regression diagnostics revealed a single, highly influential OTU (i.e., OTU 8, Proteobacteria; Suppl. Fig. 5) with a large residual (e_OTU16 $=3.24$ vs. mean_e = $-0.20)$ and Cook's D (d_OTU16 $=0.42$ vs. mean_d $=0.03$ ). After removing this single OTU from the analysis, abundance log-fold change correlated negatively (Pearson's $r=-0.54$, Spearman's rho $=-0.52$ ) with mean overall OTU abundance (Suppl. Fig. 5). This correlation, which was significant independently of the correlation test used, indicates that heat stress induces rare core OTUs to increase their abundance while the abundance of the dominant core OTUs decreases.

\section{Discussion}

Here, we provide the first characterization of the microbiome of $L$. chondrodes, a keratose sponge commonly found in salt- water aquaria around the globe [27]. With over 1300 OTUs belonging in 22 phyla, the microbial community associated with $L$. chondrodes lies well within the ranges of OTU- and phylum-level richness (i.e., 50 to 3820 OTUs, and 13 to 34 phyla, respectively) reported in a microbiome survey of 81 sponge species [33]. The high number of OTUs assigned to phylum Proteobacteria found in L. chondrodes also matches previous reports indicating that this phylum is the richest in sponge microbiomes [23]. In L. chondrodes, however, the phyla Bacteroidetes and Planctomycetes were also OTU-rich, which contrasts with findings in other sponges [23,33]. These results highlight the disparity in OTU composition displayed by sponge-associated bacterial communities and further support the proposed importance of host identity in structuring their composition [33]. Reinforcing this idea, we observed similarities between the core microbiome of cultured L. chondrodes and the most abundant bacterial OTUs obtained from a single sample of $L$. chondrodes collected in the field. Although the somewhat paradoxical scarcity of field samples of $L$. chondrodes - a species frequently found in aquaria - available for analysis precludes a conclusive assessment of the stability of the microbiome under culture 
Fig. 5 Abundance log2-fold change for core OTUs in Lendenfeldia chondrodes in thermal stress vs. control samples. The OTUs are ordered by the relative abundance with OTU 1 and OTU 388 being the OTUs with the highest and lowest abundance, respectively

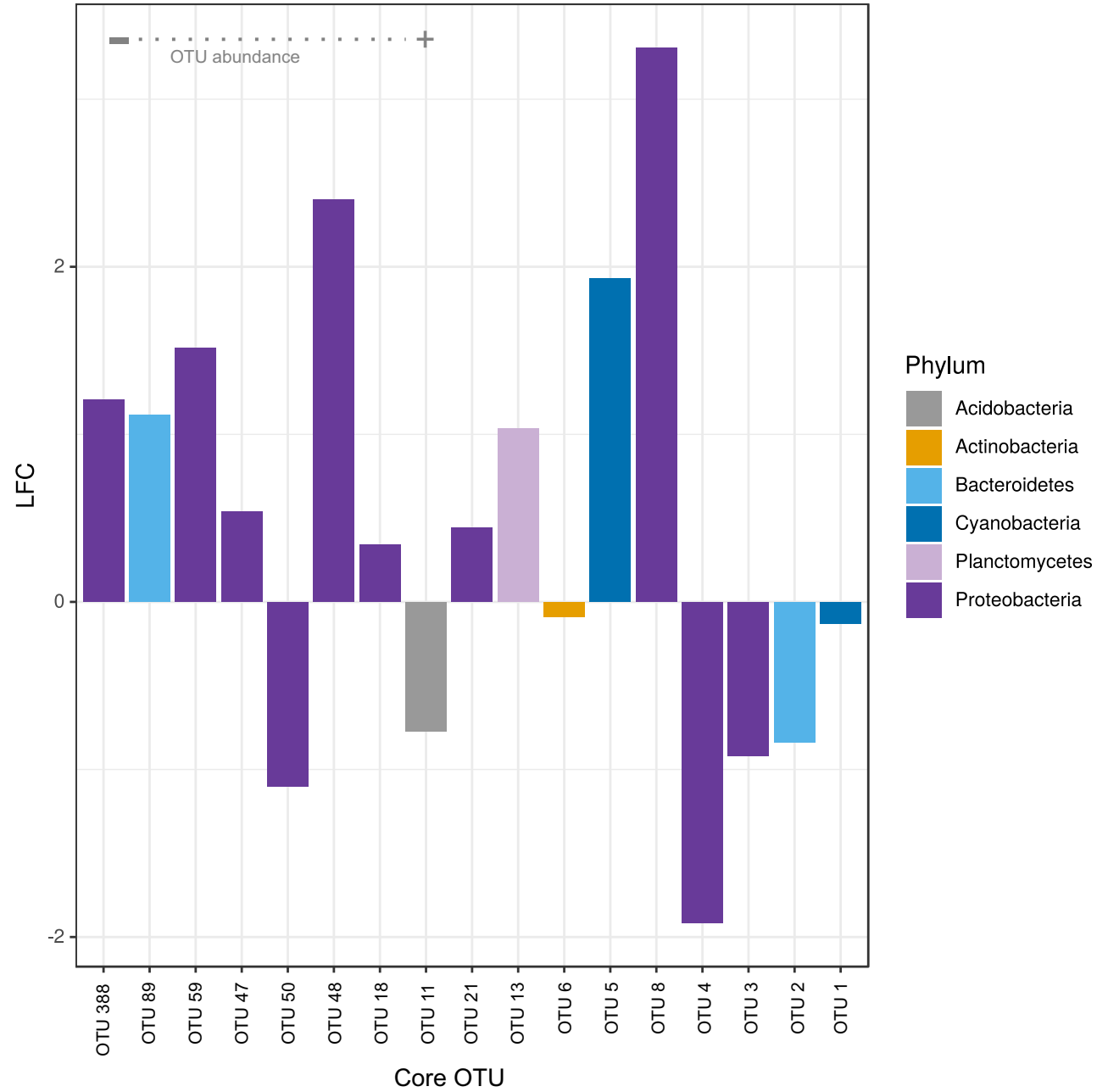

conditions in this species, our data suggest that the microbiome of $L$. chondrodes is stable under culture. We propose that this stability is driven by the dominance of a single cyanobacterium and the abundance distribution among the remaining OTUs.

Sponge microbiomes harbor a mixture of host-generalists and host-specialists and are characterized by reduced core communities of 7 to 20 OTUs [33]. The best-fitting abundance-rank dominance model for the microbiome of L. chondrodes, namely the Zipf-Mandelbrot model, allows for a similar interpretation as it points to a microbiome dominated by a small set of host-generalists, likely with few niche requirements, complemented with a low abundance but species-rich assemblage of host-specialists and transient associates with more narrow niches $[34,35]$. The original interpretation of the Zipf-Mandelbrot model associates the abundance of each item with a cost, without defining precisely what the cost is [34]. In ecology, a link between the cost of a species and its trophic level exists and implies that autotrophs cost less than heterotrophs [34]. This species-cost scheme predicts a higher abundance of autotrophs in a community, in agreement with our findings in L. chondrodes, where a single cyanobacterial OTU dominates the microbiome accounting for almost half of the $16 \mathrm{~S}$ rRNA reads sequenced. Although at present we cannot fully determine how abundance is apportioned between host- generalist and specialist bacteria in L. chondrodes, the fact that the most abundant core bacterium found in this sponge (i.e., Synechococcus spongiarum), is widespread among sponges and several other L. chondrodes' core OTUs are similar to bacteria present in other sponges point to a core microbiome largely dominated by host-generalists, as observed in other sponges [33]. It seems of interest to further investigate whether the microbiomes of other cyanosponges behave similarly and fit the Zipf-Mandelbrot model, as this model allows for mechanistic interpretations of the abundance-rank data that could provide insights into the trophic functioning of these bacterial communities.

In contrast with previous reports on several sponge species, we observed a significant compositional change in the microbiome of $L$. chondrodes upon short-term exposure to sublethal heat stress (i.e., from 27 to $31^{\circ} \mathrm{C}$ in 1 week). The Caribbean sponge Xestospongia muta responded similarly to 12 days of exposure to sublethal heat stress [8]. In both 
L. chondrodes and X. muta, the observed microbiome changes did not correlate with any signs of necrosis or discoloration of the sponge tissues. The lack of evident signs of stress at sublethal water temperatures is consistent with previous experiments in other sponge species $[4,5,10]$. However, in those species, sublethal heat stress did not trigger a significant change in the composition of the microbiome. This suggests that despite its convergent nature [33], sponge microbiomes have different levels of resilience to increasing surface seawater temperatures and can show contrasting responses to the same environmental stimulus. Thus, anthropogenicdriven climate change and the associated projected regime shifts in coral reefs [see 36,37] will result in loser and winner sponge species and will impact the composition of sponge communities in an unpredictable manner.

The response of the $L$. chondrodes microbiome to heat stress involved a re-accommodation of bacterial richness and abundance. In the case of richness, OTU-rich phyla, like Proteobacteria and Planctomycetes, became even richer after the treatment, gaining the largest number of OTUs in absolute terms during the experiment. This trend was, however, not general as other OTU-rich phyla like Bacteroidetes and Cyanobacteria showed the opposite trend, losing OTUs in response to heat stress. In relative terms, however, temperature stress had a more pronounced effect among rare phyla, with some groups doubling or halving its richness and other entirely disappearing from the community. This marked difference in the response of core and non-core OTUs to the increase in water temperature was evident in the canonical correspondence analyses done independently on these two OTU groups (see Fig. 3b). It is likely that the CCA results obtained from the analysis of the entire community are driven by the larger effect of the treatment on non-core OTUs, as rare taxa can have a high influence on the correspondence analysis results [38]. The less clear separation of control and treatment communities in the (unconstrained) NMDS analysis (Fig. 3a), which should be less affected by changes among rare OTUs, likely reflects the compositional stability of the core bacterial community of $L$. chondrodes to the treatment. Given the lack of knowledge on this symbiotic system, and generally on the specific role of the rare microbial biosphere in the functioning of the community, linking the effects of the sudden bacterial OTU gain or loss caused by heat stress with any functional changes of the $L$. chondrodes microbiome remains challenging. The development of methods to assess changes in gene expression at the holobiont level appears thus imperative to further understand L. chondrodes' interactions with its microbiome.

In sponge microbiomes, density-dependent processes determine the abundance of particular OTUs in the core community [33]. Similarly, short-term exposure to sublethal heat stress affected the abundances of core OTUs in a densitydependent manner, causing an increase and a reduction of the population size of the top four less and more abundant OTUs, respectively. The variation in abundance indicates that environmental changes can either reinforce or relax the negative feedback loop controlling the population growth of the core microbiome of $L$. chondrodes. Understanding how the interaction between the intrinsic and extrinsic population factors affects the abundance of different members of the sponge microbiome is essential to determine the effect of environmental change on the fitness of specific members of the microbiome. Establishing this link will help to provide mechanistic insights into the effects of climate change on sponge holobionts and predict their response to future oceanic conditions.

Acknowledgments We thank Monica Pichler for her constant support during the first stages of data analysis, Peter Naumann for helping set up the aquarium experiments, and Cüneyt Çaglar and Thorsten Zimmer for their support during the execution of the heat stress experiments. We also thank Lim Swee Cheng for providing access to the single specimens available of $L$. chondrodes from the wild. LL thanks Lena Wilde and David Piatka for their constant support, feedback, and motivation. LL and SV acknowledge partial funding through the Master Program in Geobiology and Paleobiology (MGAP) of the Faculty of Geosciences and the GeoBio-CenterLMU of the Ludwig-Maximilians-Universität München. GW acknowledges funding through the LMU Munich's Institutional Strategy LMUexcellent within the framework of the German Excellence Initiative. SV is indebted to N. Villalobos Trigueros, M. Vargas Villalobos, S. Vargas Villalobos, and S. Vargas Villalobos for their constant support.

Author Contributions Conceptualization: Sergio Vargas; Data curation: Laura Leiva, and Sergio Vargas; formal analysis: Sergio Vargas; funding acquisition: Sergio Vargas, Gert Wörheide; investigation: Laura Leiva; methodology: Sergio Vargas; project administration: Sergio Vargas; Resources: Sergio Vargas, Gert Wörheide; supervision: Sergio Vargas; visualization: Laura Leiva and Sergio Vargas; writing - original draft: Laura Leiva and Sergio Vargas; writing - review and editing: Sergio Vargas, Gert Wörheide.

Funding Information Open Access funding provided by Projekt DEAL.

Open Access This article is licensed under a Creative Commons Attribution 4.0 International License, which permits use, sharing, adaptation, distribution and reproduction in any medium or format, as long as you give appropriate credit to the original author(s) and the source, provide a link to the Creative Commons licence, and indicate if changes were made. The images or other third party material in this article are included in the article's Creative Commons licence, unless indicated otherwise in a credit line to the material. If material is not included in the article's Creative Commons licence and your intended use is not permitted by statutory regulation or exceeds the permitted use, you will need to obtain permission directly from the copyright holder. To view a copy of this licence, visit http://creativecommons.org/licenses/by/4.0/.

\section{References}

1. IPCC (2014) Contribution of Working Groups I, II and III to the Fifth Assessment Report of the Intergovernmental Panel on Climate 
Change. In: Core Writing Team, Pachauri RK, Meyer LA (eds) Climate Change 2014: Synthesis Report. IPCC, Geneva, p 151

2. Hughes TP, Kerry JT, Baird AH, Connolly SR, Dietzel A, Eakin CM, Heron SF, Hoey AS, Hoogenboom MO, Liu G, McWilliam MJ, Pears RJ, Pratchett MS, Skirving WJ, Stella JS, Torda G (2018) Global warming transforms coral reef assemblages. Nature 556: 492-496

3. Hughes TP, Barnes ML, Bellwood DR, Cinner JE, Cumming GS, Jackson JBC, Kleypas J, van de Leemput IA, Lough JM, Morrison TH, Palumbi SR, van Nes EH, Scheffer M (2017) Coral reefs in the Anthropocene. Nature 546:82-90

4. Webster NS, Cobb RE, Negri AP (2008) Temperature thresholds for bacterial symbiosis with a sponge. ISME J 2:830-842

5. Luter HM, Whalan S, Webster NS (2012) Thermal and sedimentation stress are unlikely causes of brown spot syndrome in the coral reef sponge, Ianthella basta. PLoS One 7:e39779

6. Massaro AJ, Weisz JB, Hill MS, Webster NS (2012) Behavioral and morphological changes caused by thermal stress in the Great Barrier Reef sponge Rhopaloeides odorabile. J Exp Mar Bio Ecol 416-417:55-60

7. Fan L, Liu M, Simister R, Webster NS, Thomas T (2013) Marine microbial symbiosis heats up: the phylogenetic and functional response of a sponge holobiont to thermal stress. ISME J 7:991-1002

8. Lesser MP, Fiore C, Slattery M, Zaneveld J (2016) Climate change stressors destabilize the microbiome of the Caribbean barrel sponge, Xestospongia muta. J Exp Mar Bio Ecol 475:11-18

9. Blanquer A, Uriz MJ, Cebrian E, Galand PE (2016) Snapshot of a bacterial microbiome shift during the early symptoms of a massive sponge die-off in the Western Mediterranean. Front. Microbiol. 7: 752

10. Simister R, Taylor MW, Tsai P et al (2012) Thermal stress responses in the bacterial biosphere of the Great Barrier Reef sponge, Rhopaloeides odorabile. Environ. Microbiol. 14:3232-3246

11. Pita L, Erwin PM, Turon X, López-Legentil S (2013) Till death do us part: stable sponge-bacteria associations under thermal and food shortage stresses. PLoS One 8:e80307

12. Strand R, Whalan S, Webster NS, Kutti T, Fang JKH, Luter HM, Bannister RJ (2017) The response of a boreal deep-sea sponge holobiont to acute thermal stress. Sci. Rep. 7:1660

13. Li CW, Chen JY, Hua TE (1998) Precambrian sponges with cellular structures. Science 279:879-882

14. Love GD, Grosjean E, Stalvies C, Fike DA, Grotzinger JP, Bradley AS, Kelly AE, Bhatia M, Meredith W, Snape CE, Bowring SA, Condon DJ, Summons RE (2009) Fossil steroids record the appearance of Demospongiae during the Cryogenian period. Nature 457: 718-721

15. Bell JJ (2008) The functional roles of marine sponges. Estuar. Coast. Shelf Sci. 79:341-353

16. Bergquist PR (2001) In: eLS (ed) Porifera (Sponges). John Wiley \& Sons, Ltd

17. JNA H, Van Soest RWM (2002) Systema porifera. A guide to the classification of sponges. In: JNA H, RWM VS, Willenz P (eds) Systema porifera. Springer US, pp 1-7

18. Taylor MW, Hill RT, Piel J, Thacker RW, Hentschel U (2007) Soaking it up: the complex lives of marine sponges and their microbial associates. ISME J 1:187-190

19. Southwell MW, Weisz JB, Martens CS, Lindquist N (2008) In situ fluxes of dissolved inorganic nitrogen from the sponge community on Conch Reef, Key Largo, Florida. Limnol Oceanogr 53:986
20. Fiore CL, Baker DM, Lesser MP (2013) Nitrogen biogeochemistry in the Caribbean sponge, Xestospongia muta: a source or sink of dissolved inorganic nitrogen? PLoS One 8:e72961

21. Muscatine L, Porter JW (1977) Reef corals: mutualistic symbioses adapted to nutrient-poor environments. Bioscience 27:454-460

22. Taylor MW, Radax R, Steger D, Wagner M (2007) Spongeassociated microorganisms: evolution, ecology, and biotechnological potential. Microbiol. Mol. Biol. Rev. 71:295-347

23. Hentschel U, Piel J, Degnan SM, Taylor MW (2012) Genomic insights into the marine sponge microbiome. Nat Rev Microbiol 10:641-654

24. Webster NS, Botté ES, Soo RM, Whalan S (2011) The larval sponge holobiont exhibits high thermal tolerance. Environ. Microbiol. Rep. 3:756-762

25. Erwin PM, Pita L, López-Legentil S, Turon X (2012) Stability of sponge-associated bacteria over large seasonal shifts in temperature and irradiance. Appl. Environ. Microbiol. 78:7358-7368

26. Morrow KM, Bourne DG, Humphrey C, Botté ES, Laffy P, Zaneveld J, Uthicke S, Fabricius KE, Webster NS (2015) Natural volcanic $\mathrm{CO} 2$ seeps reveal future trajectories for host-microbial associations in corals and sponges. ISME J 9:894-908

27. Galitz A, de C. Cook S, Ekins M et al (2018) Identification of an aquaculture poriferan "pest with potential" and its phylogenetic implications. PeerJ 6:e5586

28. Pichler M, Coskun ÖK, Ortega-Arbulú A-S et al (2018) A 16S rRNA gene sequencing and analysis protocol for the Illumina MiniSeq platform. Microbiology Open 215:e00611

29. Rognes T, Flouri T, Nichols B, Quince C, Mahé F (2016) VSEARCH: a versatile open source tool for metagenomics. PeerJ 4:e2584

30. Oksanen J, Blanchet FG, Friendly M, et al (2017) vegan: Community Ecology Package

31. Love MI, Huber W, Anders S (2014) Moderated estimation of fold change and dispersion for RNA-seq data with DESeq2. Genome Biol. 15:550

32. Wilson JB (1991) Methods for fitting dominance/diversity curves. J. Veg. Sci. 2:35-46

33. Thomas T, Moitinho-Silva L, Lurgi M, Björk JR, Easson C, Astudillo-García C, Olson JB, Erwin PM, López-Legentil S, Luter H, Chaves-Fonnegra A, Costa R, Schupp PJ, Steindler L, Erpenbeck D, Gilbert J, Knight R, Ackermann G, Victor Lopez J, Taylor MW, Thacker RW, Montoya JM, Hentschel U, Webster NS (2016) Diversity, structure and convergent evolution of the global sponge microbiome. Nat. Commun. 7:11870

34. Frontier S (1987) Applications of fractal theory to ecology. Developments in numerical ecology. Springer, Berlin Heidelberg, pp 335-378

35. Wilson JB, Wells TCE, Trueman IC et al (1996) Are there assembly rules for plant species abundance? An investigation in relation to soil resources and successional trends. J. Ecol. 84:527-538

36. Bell JJ, Davy SK, Jones T, Taylor MW, Webster NS (2013) Could some coral reefs become sponge reefs as our climate changes? Glob. Chang. Biol. 19:2613-2624

37. Bell JJ, Bennett HM, Rovellini A, Webster NS (2018) Sponges to be winners under near-future climate scenarios. Bioscience 68:955968

38. Paliy O, Shankar V (2016) Application of multivariate statistical techniques in microbial ecology. Mol. Ecol. 25:1032-1057 